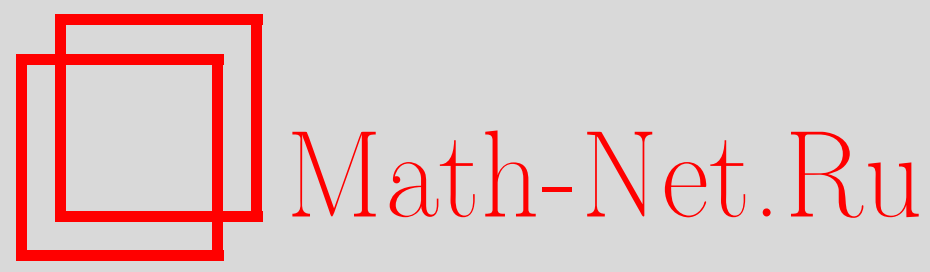

Р. Ф. Шамоян, О действии операторов свертки и Теплица в пространствах типа ВМОА, Матем. заметки, 2003, том 73, выпуск 5, 759-772

DOI: https://doi.org/10.4213/mzm222

Использование Общероссийского математического портала Math-Net.Ru подразумевает, что вы прочитали и согласны с пользовательским соглашением http://www . mathnet.ru/rus/agreement

Параметры загрузки:

IP: 35.173 .219 .12

26 апреля 2023 г., $17: 15: 24$

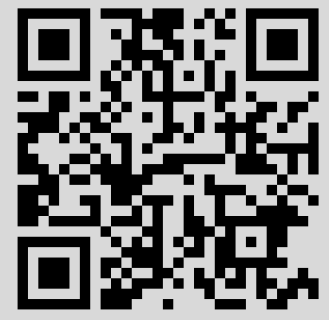




\section{О ДЕЙСТВИИ ОПЕРАТОРОВ СВЕРТКИ И ТЕПЛИЦА В ПРОСТРАНСТВАХ ТИПА ВМОА}

\section{Р. Ф. Шамоян}

В статье описаны коэффициентные мультипликаторы, исследованы действие дробной производной пространств типа ВМОА и действие операторов Теплица в этих же классах.

Библиограффия: 20 названий.

Введение. Основная цель данной заметки - распространить результаты работ [1]-[4], касаюшихся классов ВМОА, на более широкие шкалы.

Классы $\mathrm{BMOA}(U)=\mathrm{BMO}(T) \cap H^{2}(U)$ - аналитические подпространства классов функций с ограниченной средней осцилляцией - играют важную роль в различных вопросах теории пространств аналитических функций (см., например, [5]).

В работе автора [6] были введены и исследованы некоторые свойства классов типа ВМОА. Отправной точкой для определения этих пространств была известная норма Гарсиа для $\mathrm{BMO}(T)$ (см., например, [5]).

В этой работе мы продолжим изучение пространств типа ВМОА в единичном круге $U$, при этом расширение шкалы классов ВМОА ниже “опирается" не только на вьше упомянутую норму Гарсиа, но и на следующий классический результат теории ограниченных аналитических функций (см. [5]): $f \in$ ВМОА в том и только в том случае, когда мера $\mu(z)=\left|f^{\prime}(z)\right|^{2}(1-|z|)$ является мерой Карлесона.

Для формулировки утверждений нам понадобится ряд стандартных обозначений.

Пусть $U$ - единичный круг на комплексной плоскости $\mathbb{C} ; U=\{z:|z|<1\}, T=$ $\{z:|z|=1\}$ - его граница, $H(U)$ - класс всех голоморфных в $U$ функций, $d m(\xi)$ и $d m_{2}(z)$ - нормированные меры Лебега на $T$ и в $U$ соответственно, $H^{p}(U)$ - классы Харди в круге $U$ (см., например, [5]), $M_{p}^{p}(f, r)=\int_{T}|f(r \xi)|^{p} d m(\xi)$.

Введем пространства типа ВMOA: $F_{s}^{\infty, q}(U)\left(\right.$ см. [7]) и $\mathrm{BMOA}_{\alpha, n, q, \beta}(U)$. Имеем

$$
\begin{aligned}
F_{s}^{\infty, q}(U)= & \left\{f \in H(U): \sup _{\omega \in U}\left(\int_{U} \frac{\left|\widetilde{D}^{k} f(z)\right|^{q}(1-|z|)^{(k-s) q-1}}{|1-z \bar{\omega}|^{2+N}} d m_{2}(z)\right)(1-|\omega|)^{N}<\infty,\right. \\
& \text { где } k \text { любое, } k \in \mathbb{N}, k>s, s \in \mathbb{R}, q \in(0, \infty)\},
\end{aligned}
$$

Работа поддержана Российским фондом фундаментальных исследований, грант № 01-01-00992. 
где $N \in \mathbb{N}, N$ - некоторое фиксированное число,

$$
\begin{aligned}
\operatorname{BMOA}_{\alpha, n, q, \beta}= & \left\{f^{(n)} \in H^{1}(U): \sup _{|z|<1} \int_{T} \frac{\left|\widetilde{D}^{\alpha} f(\xi)-\widetilde{D}^{\alpha} f(z)\right|}{|1-\bar{\xi} z|^{q}} d m(\xi)(1-|z|)^{\beta}<\infty,\right. \\
& \text { где } \alpha, \beta \geqslant 0, q \in(0, \infty), n \in \mathbb{N}\},
\end{aligned}
$$

где $\widetilde{D}^{\alpha}-$ дифференциальный оператор

$$
\widetilde{D}^{\alpha}: H(U) \rightarrow H(U):\left(\widetilde{D}^{\alpha} f\right)(z)=\sum_{k \geqslant 0}(k+1)^{\alpha} a_{k} z^{k}, \quad f(z)=\sum_{k \geqslant 0} a_{k} z^{k} \alpha \in \mathbb{R}
$$

Учитывая определения пространств ВМОА через норму Гарсиа и через меры Карлесона легко видеть, что $F_{0}^{\infty, 2}(U)=\mathrm{BMOA}_{0,0,2,1}=\mathrm{BMOA}$ (см. [5], [8]). Классы $\mathrm{BMOA}_{\alpha, n, q, \beta}$ были рассмотрены в [6] (см. также [9]). Пространства $F_{s}^{\infty, q}$ в единичном шаре $B^{n}$ пространства $C^{n}$ были введены, по-видимому, впервые в статье [7]. В $\mathbb{R}^{n}$ их аналоги изучены в монографии [10]. В [7] классы $F_{s}^{\infty, q}$ возникают при описании пространств линейных непрерьвных функционалов голоморфных классов типа Лизоркина-Трибеля. Тем самым, классы $F^{\infty, q}$, с одной стороны, расширяют шкалу пространств ВМОА, а с другой - являются “предельной точкой" интенсивно изучающихся в настоящее время голоморфных классов типа Лизоркина-Трибеля (см. [7], [9], [11], [12]).

1. О действии операторов свертки в пространствах $F_{s}^{\infty, q}$. Всюду ниже в работе через $C, C^{\prime}, C(\alpha), C(\alpha, q)$ мы обозначаем различные положительные константы, зависящие от $\alpha, q$ и т.д., а запись $A \lesssim B$ означает, что существует положительная константа $C$ такая, что $A \leqslant C B$. Все вспомогательные леммы, использующиеся при установлении результатов заметки, собраны в последнем третьем разделе работы.

Далее, как обьчно (см., например, [1]), скажем, что последовательность $\left(c_{k}\right)$ определяет ограниченный оператор свертки, действующий из $X$ в $Y(X, Y$-подпространства $H(U))$ или является мультипликатором из $X$ в $Y$, если для любой функции $f, f \in X$, $f(z)=\sum_{k \geqslant 0} a_{k} z^{k}$, функция $g(z)=\sum_{k \geqslant 0} c_{k} a_{k} z^{k}$ лежит в $Y$. Множество всех таких $\left\{\left(c_{k}\right)\right\}$ мы обозначим через $\widetilde{M_{T}}(X, Y)$.

Введем еще один дифференциальный оператор, которьй потребуется нам в дальнейшем $D^{\alpha}: H(U) \rightarrow H(U)$,

$$
\left(D^{\alpha} f\right)(z)=\sum_{k \geqslant 0} \frac{\Gamma(k+\alpha+1)}{\Gamma(\alpha+1) \Gamma(k+1)} a_{k} z^{k}, \quad f=\sum_{k \geqslant 0} a_{k} z^{k}, \quad \alpha>-1 .
$$

Легко видеть, что

$$
D^{\alpha}\left(\frac{1}{1-z}\right)=\frac{1}{(1-z)^{\alpha+1}}, \quad \alpha>-1, \quad z \in U
$$

В дальнейшем $A_{\alpha}^{p}(U)$ - хорошо известные классы Бергмана в круге $U$ (см. [13]). 
TeOPEMA 1. 1) Пyсmb $s>0, q \geqslant 2 ;$ morda

$\widetilde{M_{T}}\left(H^{1}(U), F_{s}^{\infty, q}(U)\right)=\left\{g \in H(U): \sup _{|z|<1} M_{\infty}\left(\widetilde{D}^{k} g,|z|\right)(1-|z|)^{k-s}<\infty\right\}=H_{k, k-s}^{\infty, \infty}$,

где $k$ любое, $k \in \mathbb{N}, k>s, g \sim\left(c_{k}\right)$.

2) Пусть $q \geqslant 1, \alpha>-1$; тогда

$$
\begin{aligned}
\widetilde{M_{T}}\left(A_{\alpha}^{1}(U), F_{s}^{\infty, q}(U)\right) & =\left\{g \in H(U): \sup _{|z|<1} M_{\infty}\left(\widetilde{D}^{k} g,|z|\right)(1-|z|)^{k-s-(\alpha+1)}<\infty\right\} \\
& =H_{k, k-s-(\alpha+1)}^{\infty, \infty}
\end{aligned}
$$

где $k$ любое, $k \in \mathbb{N}, k>s+(\alpha+1)+q, g \sim\left(c_{k}\right)$.

ЗАмЕчаниЕ 1. Учитывая определение пространств ВМОА через меры Карлесона, имеем $F_{0}^{\infty, 2}=$ ВМОА. Описание пространства $\overline{M_{T}}\left(H^{1}, F_{0}^{\infty, 2}\right)=\widehat{M_{T}}\left(H^{1}, \mathrm{BMOA}\right)$ получено в работе [1] и совпадает с описанием, приведенньм в сформулированной вьше теореме. Учитывая лемму 2 (см. п. 3), всюду в теореме 1 оператор $\widetilde{D}^{\alpha}$ можно заменить оператором $D^{\alpha}$.

ЗАмЕчАнИЕ 2. Изучение пространств коэффициентных мультипликаторов ВМОА было, по-видимому, начато в работе [1]. В [14] дано описание классов $\widetilde{M_{T}}(B \ell, \mathrm{BMOA})$ и $\widetilde{M_{T}}\left(\Lambda_{A}^{\alpha}, \mathrm{BMOA}\right)$, где $B \ell$ и $\Lambda_{A}^{\alpha}$ - известные классы Блоха и Лишшица-Зигмунда соответственно.

ДокАЗАТЕЛЬСТво тЕоремы 1. Докажем сначала включение $\widetilde{M_{T}}\left(H^{1}, F_{s}^{\infty}, q\right) \subset$ $H_{k, k-s}^{\infty, \infty}(U)$. Для этого необходимо использовать стандартные рассуждения (см., например, [12], [11]), теорему о замкнутом графике и пробную функцию

$$
f_{r}(z)=\frac{1}{(1-r z)^{\ell+1}}=\sum_{k \geqslant 0} a_{k} z^{k}=D^{\ell}\left(\frac{1}{1-r z}\right), \quad r \in I, \quad z \in U, \quad \ell \in \mathbb{N} .
$$

Имеем

$$
\left\|D^{\ell} g_{r}\right\|_{F_{s}^{\infty, q}} \lesssim\left\|\frac{1}{(1-r z)^{\ell+1}}\right\|_{H^{1}} \lesssim \frac{1}{(1-r)^{\ell}}
$$

где $g \in \widetilde{M_{T}}\left(H^{1}, F_{s}^{\infty, q}\right)$.

Учтем, что из леммы 1 следует оценка

$$
\left|D^{\ell} D^{k} g_{r}(z)\right|(1-|z|)^{k-s} \lesssim\left\|D^{\ell} g_{r}\right\|_{F_{s}^{\infty, q}}, \quad 0<q<\infty, \quad k>s, \quad \ell \in \mathbb{N}, \quad z \in U .
$$

Положим $z=r \xi ; \quad$ тогда $\sup _{z}\left|\widetilde{D}^{\ell+k} g(z)\right|(1-|z|)^{\ell+k-s}<\infty ; \quad$ следовательно, $\sup _{z}\left|\widetilde{D}^{k} g(z)\right|(1-|z|)^{k-s}<\infty, k>s$.

Для доказательства обратного включения воспользуемся следующей оценкой, установленной в [7]:

$$
\|f\|_{F_{s}^{\infty, q}} \lesssim \int_{0}^{1}\left(M_{\infty}\left(D^{\left[s^{+}\right]} f, r\right)\right)^{q}(1-r)^{\left(\left[s^{+}\right]-s\right) q-1} d r,
$$


где $\left[s^{+}\right]=[s+1]$.

Оценка (1) установлена для дифференциальных операторов $\widetilde{D}^{\alpha}$; в силу результатов работы [15] она остается справедливой и для операторов $D^{\alpha}$.

Пусть $g \in H_{k, k-s}^{\infty, \infty}, g(z)=\sum_{k \geqslant 0} c_{k} z^{k}, f \in H^{1}, f(z)=\sum_{k \geqslant 0} a_{k} z^{k}, h(z)=$ $\sum_{k \geqslant 0} c_{k} a_{k} z^{k}$. Тогда

$$
\left|h\left(r^{2} \xi\right)\right|=\left(\frac{1}{2 \pi}\right)\left|\int_{T} g(r t \xi) f(r \bar{t}) d m(t)\right| .
$$

Отсюда, учитьвая, что $g \in H_{k, k-s}^{\infty, \infty}$, где $k$ подобрано так, что $s<k$, получаем

$$
\left|\widetilde{D}^{s+k} h\left(r^{2} \xi\right)\right|^{q}(1-r)^{k q-1} \lesssim\left(M_{1}\left(\widetilde{D}^{s} f, r\right)\right)^{q}(1-r)^{(s-k) q}(1-r)^{k q-1} .
$$

Следовательно, опираясь на легко выводимую оценку из леммы 1

$$
\begin{gathered}
\left(\int_{0}^{1} M_{1}(G, R)(1-R)^{\alpha} d R\right)^{p} \leqslant\left(\int_{0}^{1}\left(M_{1}(G, R)\right)^{p}(1-R)^{\alpha p+p-1} d R\right), \\
0<p \leqslant 1, \quad \alpha>-1, \quad G \in H(U),
\end{gathered}
$$

Выводим

$$
\int_{0}^{1}\left(M_{\infty}\left(\widetilde{D}^{s+k} h, r\right)\right)^{q}(1-r)^{k q-1} d r \lesssim\left(\int_{0}^{1}\left(M_{1}\left(\widetilde{D}^{s} f, r\right)\right)^{2}(1-r)^{2 s-1} d r\right)^{\frac{q}{2}} \lesssim\|f\|_{H^{1}}^{q} .
$$

В последнем неравенстве мы воспользовались известной теоремой Харди-Литтлвуда (см. [15]). Остается еще раз использовать теорему Харди-Литтлвуда и оценки (1):

$$
\|h\|_{F_{s}^{\infty, q}}^{q} \lesssim \int_{0}^{1}\left(M_{\infty}\left(\widetilde{D}^{s+1} h, r\right)\right)^{q}(1-r)^{q-1} d r \lesssim \int_{0}^{1}\left(M_{\infty}\left(\widetilde{D}^{s+k} h, r\right)\right)^{q}(1-r)^{k q-1} d r .
$$

Докажем только включение $H_{k, k-s-(\alpha+1)}^{\infty, \infty} \subset \widetilde{M_{T}}\left(A_{\alpha}^{1}, F_{s}^{\infty, q}\right)$. Обратное устанавливается совершенно аналогично предыдущему случаю.

Рассуждая так же, как и в предыдущем случае, получаем по лемме 2

$$
\left|D^{s+1} h_{|\widetilde{\omega}|}(z)\right| \lesssim \int_{U}\left|D^{1+s} D^{\ell} D^{-k} f(\tilde{z})\right| \cdot\left|D^{k} g_{|\widetilde{\omega}|}(\tilde{z})\right|(1-|\tilde{z}|)^{\ell-1} d m_{2}(\tilde{z}),
$$

где $\max \{k-s-\alpha-2,1\}<\ell<(k-s-\alpha-1)$.

Далее, $(1-|\tilde{z}|)^{\ell-1} \leqslant(1-|\tilde{z}||\widetilde{\omega}|)^{\ell-1},(1-|\tilde{z}||\widetilde{\omega}|)^{\ell-1}=(1-|\tilde{z}||\widetilde{\omega}|)^{k-s-(\alpha+1)} \times$ $(1-|\tilde{z}||\widetilde{\omega}|)^{\alpha+s-k+\ell}$. Положим $z=\widetilde{\omega}$.

Учитывая, что $g \in H_{k, k-s-(\alpha+1)}^{\infty, \infty}$, оценку

$$
\int_{0}^{1}(1-|\tilde{z}||\widetilde{\omega}|)^{\alpha+s-k+\ell}(1-|\widetilde{\omega}|)^{v-1} d|\widetilde{\omega}| \lesssim(1-|\tilde{z}|)^{\alpha+s-k+\ell+v}
$$


при $v=1, k-s-\alpha-q>\ell>1$, и (10), получим

$$
\begin{aligned}
\|f\|_{F_{s}^{\infty}, q} & \lesssim\left(\int_{0}^{1}\left(M_{\infty}\left(D^{s+1} h,|\widetilde{\omega}|\right)\right)^{q}(1-|\widetilde{\omega}|)^{q-1} d|\widetilde{\omega}|\right)^{\frac{1}{q}} \\
& \lesssim \int_{U}\left|D^{\ell} D^{-k} D^{s+1} f(\tilde{z})\right| \int_{0}^{1}(1-|\widetilde{\omega}|)^{\ell-1}(1-|\tilde{z}||\widetilde{\omega}|)^{\alpha+s-k+\ell} d|\widetilde{\omega}| d m_{2}(\tilde{z}) \\
& \lesssim \int_{U}|f(\tilde{z})|(1-|\tilde{z}|)^{\alpha} d m_{2}(\tilde{z}), \quad q \geqslant 1 ;
\end{aligned}
$$

в последнем неравенстве мы использовали теорему Харди-Литтлвуда (см. [15]) и оценкy (2).

Теорема 1 доказана полностью.

Нам потребуется следующее определение. Пусть $X$ - некоторое подпространство $H(U)$. Скажем, что ряд Тейлора сходится в пространстве $X$, если для любой функции $f$ из $X: f(z)=\sum_{k=0}^{\infty} a_{k} z^{k},\left\|S_{n}-f\right\|_{X} \rightarrow 0$ при $n \rightarrow \infty$, где $S_{n}(z)=\sum_{k=0}^{n} a_{k} z^{k}$.

Первая часть следуюшего утверждения установлена в [3] (см. также [16]), вторая доказана в [2].

Tеорема А. 1) Пусть $p>1, f^{\prime} \in A_{p-2}^{p}$; тогда $f \in$ BMOA.

2) В пространстве ВМОА существует функиия $f$ такая, что ее ряд Тейлора не сходится по норме ВMOA.

Ниже мы приводим обобщение теоремы А в нескольких направлениях. Пусть

$$
\begin{gathered}
H_{\gamma}^{p, q, \alpha}=\left\{f \in H(U): \int_{0}^{1} M_{p}^{q}\left(\widetilde{D}^{\gamma} f, r\right)(1-r)^{\alpha q-1} d r<\infty,\right. \\
0<p, q \leqslant \infty, \quad \gamma \in \mathbb{R}, \quad \alpha \in(0, \infty)\}
\end{gathered}
$$

(cм. [17]), $H_{0}^{p, p,(\alpha+1) / p}=A_{\alpha}^{p}$.

Tеорема 2. Если $g(z) \in H_{\alpha+1-s}^{q, \infty,(\alpha+1) / q}, \quad q \in(1, \infty), \quad \alpha>-1, \quad s \in \mathbb{R}, \quad g(z)=$ $\sum_{k \geqslant 0} c_{k} z^{k}$, то последовательность $\left\{\left(c_{k}\right)_{k \geqslant 0}\right\}-$ мультипликатор из $A_{\alpha}^{p}$ в $F_{s}^{\infty, \nu}$, где $1 / p+1 / q=1,1 / \nu+1 / \ell=1$.

ДоКАЗАТЕЛЬСТво ТЕОРЕМЫ 2. Заметим сначала, что неравенство $\|f\|_{\text {ВмоА }}=$ $\|f\|_{F_{0}^{\infty, 2}} \lesssim\left\|f^{\prime}\right\|_{A_{p-2}^{p}}$ равносильно оценке

$$
\left\|\widetilde{D}^{-1} f\right\|_{F_{0}^{\infty, 2}} \lesssim\|f\|_{A_{p-2}^{p}}, \quad \text { где }\left(\widetilde{D}^{-1} f\right)(z)=\sum_{k \geqslant 1}\left(\frac{a_{k}}{k+1}\right) z^{k} .
$$

Нам понадобится следующее утверждение, установленное в работе [17]:

$$
\widetilde{M_{T}}\left(H^{p, \nu, \alpha}, H^{q, q, \beta}\right)=H_{\alpha+\frac{1}{p}-1}^{q, \nu * q, \beta}, \quad \text { где } \quad p \leqslant 1, \quad p \leqslant q \leqslant \infty, \quad 0<\alpha, \quad \beta<\infty
$$

$(\nu * q)^{-1}=1 / q-1 / \nu$ при $q<\nu$ и $\nu * q=\infty$ при $\nu \leqslant q$. 
Опираясь на это утверждение, несложно вьписать достаточные условия для того, чтобы последовательность $\left\{\left(c_{k}\right)\right\}\left(g(z)=\sum_{k \geqslant 0} c_{k} z^{k}\right)$ была мультипликатором из $H_{\left[s^{+}\right]}^{1, \ell,\left[s^{+}\right]-s}$ в пространства $H_{\alpha+1}^{q, q,(\alpha+1) / q}$, где $\left[s^{+}\right]$- целая часть числа $s+1, \ell, q \in(1, \infty)$, $\alpha>-1$.

Достаточно заметить, что если $g$ - мультипликатор из $X$ в $Y(X, Y \subset H(U)$ - некоторые подпространства), то $D^{-\alpha} D^{\beta} g$ - мультипликатор из $D^{\alpha} X$ в $D^{\beta} Y$, где $D^{\alpha} X=$ $\left\{f \in H(U): D^{\alpha} f \in X\right\}$.

Поэтому выводим

$$
\begin{aligned}
& \widetilde{M_{T}}\left(H_{\left[s^{+}\right]}^{1, \ell,\left[s^{+}\right]-s}, H_{\alpha+1}^{q, q,(\alpha+1) / q}\right)=H_{\alpha+1-s}^{q, \infty,(\alpha+1) / q} \text { при } \ell \leqslant q, \\
& \widetilde{M_{T}}\left(H_{\left[s^{+}\right]}^{1, \ell,\left[s^{+}\right]-s}, H_{\alpha+1}^{q, q,(\alpha+1) / q}\right)=H_{\alpha+1-s}^{q, \ell * q,(\alpha+1) / q} \text { при } \quad \ell>q
\end{aligned}
$$

(последнее равенство выписано для полноты изложения).

Далее, из неравенства Минковского следует, что

$$
F_{s}^{1, \ell} \subset H_{\left[s^{+}\right]}^{1, \ell,\left[s^{+}\right]-s}, \quad \ell \in(1, \infty), \quad s \in \mathbb{R},
$$

где (см. [7])

$$
F_{s}^{q, \ell}=\left\{f \in H(U): \int_{T}\left(\int_{0}^{1}\left|\widetilde{D}^{\left[s^{+}\right]} f(r \xi)\right|^{\ell}(1-r)^{\ell\left(\left[s^{+}\right]-s\right)-1} d r\right)^{q / \ell} d m(\xi)<\infty\right\}
$$

$\ell, q \in(0, \infty), s \in \mathbb{R}$.

Учитывая, что

$$
\left(H_{\alpha+1}^{q, q,(\alpha+1) / q}\right)^{*}=H_{0}^{p, p,(\alpha+1) / p}=A_{\alpha}^{p}, \quad\left(F_{s}^{1, \ell}\right)^{*}=F_{s}^{\infty, \nu}, \quad \frac{1}{p}+\frac{1}{q}=1, \quad \frac{1}{\ell}+\frac{1}{\nu}=1
$$

(см. [16] и [7] соответственно), $X^{*}$ - сопряженное к $X$ пространство, и опираясь на соображения двойственности, получаем, что условия, указанные в теореме, будут также достаточньми для того, чтобы последовательность $\left\{\left(c_{k}\right)\right\}\left(g(z)=\sum_{k \geqslant 0} c_{k} z^{k}\right)$ была мультипликатором из $H_{0}^{p, p, \frac{\alpha+1}{p}}$ в $F_{s}^{\infty, \nu}$. Теорема установлена при $q \geqslant \ell$.

Для доказательства теоремы 2 при $q<\ell$ проводим аналогичные рассуждения. Вместо вложения (5) используем вложение $F_{s}^{1, \ell} \subset H_{\left[t^{+}\right]}^{r, 1,\left[t^{+}\right]-t}($ см. [7]), $s-1=t-1 / r$, $\ell, r>1$.

Далее, из равенства $\left(2^{\prime}\right)$ получаем достаточное условие на последовательность $\left\{\left(c_{k}\right)\right\}$ такую, что $\left\{\left(c_{k}\right)\right\}\left(g(z)=\sum_{k \geqslant 0} c_{k} z^{k}\right)$ - мультипликатор из $H_{\left[t^{+}\right]}^{r, 1,\left[t^{+}\right]-t}$ в $H_{\alpha+1}^{q, q,(\alpha+1) / q}$, а значит, $\left\{\left(c_{k}\right)\right\} \in \widetilde{M_{T}}\left(F_{s}^{1, \ell}, H_{\alpha+1}^{q, q,(\alpha+1) / q}\right) \subset \widetilde{M_{T}}\left(A_{\alpha}^{p}, F_{s}^{\infty, \nu}\right), 1 / \nu+1 / \ell=1$, т.е. $g(z) \in$ $H_{\alpha+1-s}^{q, \infty,(\alpha+1) / q}$. Теорема установлена.

ЗАмЕчАниЕ 3 . Легко видеть, что для получения утверждения п. 1, содержашегося в теореме А, необходимо положить $\ell=2, s=0, \alpha=p-2$ в теореме 2 , учесть, что $F_{0}^{\infty, 2}=$ BMOA, и заметить, что при вьшеуказанных $\ell, s$ и $\alpha$ функция $g(z)=\sum_{k \geqslant 0} z^{k} /(k+1)$ по лемме 1 из класса $H_{p-1}^{q, \infty,(p-1) / q}, 1 / p+1 / q=1$, т.е. мультипликатор. 
Теорема 3. 1) При $t<0, \quad \ell \in(0, \infty)$ ряды Тейлора сходятся по норме в пространстве $F_{t}^{\infty, \ell}$.

2) Для любой функиии $f, f(z)=\sum_{k \geqslant 0} f_{k} z^{k}, f \in F_{t}^{\infty, \ell}$, имеют место соотношения

$$
\begin{aligned}
& \lim _{n \rightarrow \infty}\left\|D^{-\alpha} \sum_{k=n}^{\infty} f_{k} z^{k}\right\|_{F_{t}^{\infty, \ell}}=0 \quad \text { при любом } \quad \ell \geqslant 1, \quad \alpha>0, \quad t \in \mathbb{R}, \\
& \lim _{n \rightarrow \infty}\left\|D^{-\alpha} \sum_{k=n}^{\infty} f_{k} z^{k}\right\|_{F_{t}^{\infty, \ell}}=0 \quad \text { при любом } \quad \ell<1, \quad \alpha>\frac{1}{\ell}-1, \quad t \in \mathbb{R} .
\end{aligned}
$$

ЗАмЕчАниЕ 4. Легко видеть, что утверждения теоремы 3 распространяют результат п. 2) теоремы А. Это следует сразу из равенства $F_{0}^{\infty, 2}=$ BMOА.

ДокАЗАТЕЛЬСтво тЕОремы 3. В пространствах $A_{\alpha}^{p}, p \in(1, \infty), \alpha \in(-1, \infty)$, ряды Тейлора сходятся по норме. Это установлено в [2].

Заметим, что (см. [7])

$$
\left\|f_{n}-f\right\|_{F_{t}^{\infty, \ell}} \leqslant\left\|f_{n}-f\right\|_{F_{s}^{p, p},}, \quad p<\infty, \quad s-\frac{1}{p}=t, \quad s \in \mathbb{R}, \quad \ell \in(0, \infty) .
$$

Из этого соотношения и теоремы Харди-Литтлвуда (см. [15]) выводим

$$
\begin{gathered}
\left\|f_{n}-f\right\|_{F_{t}^{\infty, \ell}} \lesssim \int_{U}\left|\left(f_{n}-f\right)(z)\right|^{p}(1-|z|)^{-\left(t+\frac{1}{p}\right) p-1} d m_{2}(z), \quad \ell \in(0, \infty) \\
p \in(1, \infty), \quad t \in \mathbb{R}, \quad t+\frac{1}{p}<0
\end{gathered}
$$

Следовательно, если $t<-1 / p$ (т.е. $t<0)$, то при любом $\ell \in(0, \infty)$ ряды Тейлора сходятся по норме в $F_{t}^{\infty, \ell}$. При $\ell>1$ это можно показать также следующим образом:

$$
\begin{aligned}
\left\|f_{n}-f\right\|_{F_{t}^{\infty, \ell}} & \lesssim\left\|f_{n}-f\right\|_{H_{\left[t^{+}\right]}^{\infty, \ell,\left[t^{+}\right]-1}} \lesssim\left\|f_{n}-f\right\|_{\left.H_{\left[s^{+}\right]}^{\ell, \ell,[s}\right]-s} \\
& \lesssim \int_{U}\left|\left(f_{n}-f\right)(z)\right|^{\ell}(1-|z|)^{-s \ell-1} d m_{2}(z), \\
s & -\frac{1}{\ell}=t, \quad \ell \in(1, \infty), \quad s<0 ;
\end{aligned}
$$

первые вложения установлены в [7], последнее - следствие теоремы Харди-Литтлвуда.

Поэтому при $t+1 / \ell<0, \ell>1$ (т.е. при $t<-1 / \ell, \ell>1$ ) в $F_{t}^{\infty, \ell}$ ряды Тейлора сходятся по норме. Отметим также, что можно опереться на соображения двойственности, используемые в [2] и вьвести сходимость рядов Тейлора при $\ell>1, s<0$ в $F_{s}^{\infty, \ell}$ из равенства $\left(F_{s}^{1, q}\right)^{*}=F_{s}^{\infty, \ell}, s \in \mathbb{R}, 1 / \ell+1 / q=1$ и оценок

$$
\left\|f_{n}-f\right\|_{F_{s}^{1, q}} \lesssim I(n)=\int_{T} \int_{0}^{1}\left|\left(f_{n}-f\right)(z)\right|^{q}(1-|z|)^{-q s-1} d|z| d m(\xi), \quad s<0
$$

(см. [7]). Далее, учитываем, что $I=I(n) \rightarrow 0$ при $n \rightarrow \infty$ (см. [2]). 
Займемся доказательством второго утверждения в теореме. Из леммы 2 (п. 1) вьводим

$$
\left|\widetilde{D}^{k} D^{-\alpha} h(z)\right| \lesssim \int_{U}\left|\widetilde{D}^{k} f_{z}(\bar{\varphi})\right|\left|D^{\alpha} D^{-\alpha} g_{|\widetilde{\omega}|}(\widetilde{\omega})\right|(1-|\widetilde{\omega}|)^{\alpha-1} d m_{2}(\widetilde{\omega}),
$$

$\widetilde{\omega}=\varphi|\widetilde{\omega}|$, где

$$
\begin{gathered}
g(\widetilde{\omega})=g(n, \widetilde{\omega})=\widetilde{\omega}^{n+1}(1-\widetilde{\omega}), \quad h(z)=\sum_{k=n+1}^{\infty} \hat{f}(k) z^{k}, \\
f(z)=\sum_{k=0}^{\infty} \hat{f}(k) z^{k}, \quad f \in F_{s}^{\infty, \ell}, \\
h\left(z r^{2}, n\right)=h\left(z r^{2}\right)=\left(\frac{1}{2 \pi}\right) \int_{T} f_{z}(r \bar{t})\left((r t)^{n+1}+(r t)^{n+2}+\cdots\right) d m(t),
\end{gathered}
$$

$f_{z}(\omega)=f(z \omega), z, \omega \in U, \alpha>0, r>1 / 2$.

Далее, учитьвая вид “нормы" в классах $F_{s}^{\infty, \ell}$, из (6) нетрудно получить оценку

$$
\left\|D^{-\alpha} h(z)\right\|_{F_{s}^{\infty, \ell}}^{\ell} \lesssim\|f\|_{F_{s}^{\infty, \ell}}^{\ell}\left(\int_{U}|g(n, \widetilde{\omega})|(1-|\widetilde{\omega}|)^{\alpha-1} d m_{2}(\widetilde{\omega})\right)^{\ell}, \quad \ell>1,
$$

и из леммы 1 (п. 4)

$$
\left\|D^{-\alpha} h(z)\right\|_{F_{s}^{\infty, \ell}}^{\ell} \lesssim\|f\|_{F_{s}^{\infty, \ell}}^{\ell} \int_{U}|g(n, \widetilde{\omega})|^{\ell}(1-|\widetilde{\omega}|)^{\alpha \ell+\ell-2} d m_{2}(\widetilde{\omega}), \quad \ell \leqslant 1 .
$$

Остальное ясно. Теорема 3 доказана.

2. Операторы Теплица и деление на внутреннюю функцию в классах типа ВМОА. В этом разделе мы установим утверждение о делении на внутренний множитель в пространствах типа ВМОА. Напомним следующее определение (см. [18]).

Пусть $X$ - подкласс класса $H^{1}(U)$. Скажем, что в $X$ возможнн деление на внутренний множитель, если для любой функции $g \in X$ и для любой внутренней функции $f$ (см. [5]) имеет место импликация $B_{g} S_{g} f^{-1} \in H^{\infty} \Rightarrow g f^{-1} \in X$, где $B_{g}, S_{g}$ - соответственно произведение Бляшке и сингулярная внутренняя функция, соответствующая функции $g$ (см. [5]).

Деление на внутренний множитель в различных пространствах голоморфных в $U$ функций устанавливалось в ряде работ различных авторов (см. [18] и указанную там литературу). В ВМОА это сделать нельзя, однако, как было установлено в [4], в пространствах $\mathrm{BMOA}_{n}=\left\{f \in H(U): \widetilde{D}^{n} f \in \mathrm{BMOA}, n \in \mathbb{N}\right\}$ функции делятся на свою внутреннюю часть. Доказательство этого факта было основано на соображениях двойственности и на одной новой характеристике ВМОА, вывод которой, в свою очередь, существенным образом опирался на "представление Феффермана" классов ВМО (см. [5]).

Для получения обобщения этого результата на гладкостные аналоги пространств типа ВМОА, мы предложим подход, в котором основную роль будет играть характеристика пространств ВМОА через норму Гарсиа (см. [8], [5]):

$$
\mathrm{BMOA}=\left\{f \in H^{1}(U): \sup _{|z|<1} \int_{T} \frac{|f(\xi)-f(z)|^{s}}{|1-\bar{\xi} z|^{2}} d m(\xi)(1-|z|)<\infty, \quad 1 \leqslant s<\infty\right\} .
$$


Напомним, что

$$
\mathrm{BMOA}_{\alpha, n, q, \beta}=\left\{f^{(n)} \in H^{1}: \sup _{|z|<1} \int_{T} \frac{\left|\widetilde{D}^{\alpha} f(\xi)-\widetilde{D}^{\alpha} f(z)\right|}{|1-\bar{\xi} z|^{q}}(1-|z|)^{\beta} d \xi<\infty\right\},
$$

где $0 \leqslant \alpha, q, \beta<\infty, n \in \mathbb{N}$.

ТЕОрема 4. Пусть выполнена иепочка неравенств $\beta+1<q<\beta+2, n \in \mathbb{N}$, $\beta>0, \alpha \geqslant 0$. Тогда в классах $\mathrm{BMOA}_{\alpha, n, q, \beta}(U)$ возможнно деление на внутреннюю функиию.

ЗАмЕчаниЕ 5 . При $\alpha=n \in \mathbb{N}, q=2, \beta=1$ этот результат, и об этом было упомянуто вьше, был установлен в [4]; при $\alpha=n=0, q=2, \beta=1$ теорема не верна (см. [4]).

ЗАмечаниЕ 6. Действие оператора Теплица и неразрывно связанных с ним операторов Ганкеля в классах ВМОА изучалось в [19], [20] (см., также [11]).

ЗАмЕчАниЕ 7. Из теоремы 4 следует, что эффект деления на внутренний множитель может быть сохранен и при устранении гладкости, но за счет “деформации" ядра Пуассона (случай $\alpha=0)$.

ДОКАЗАТЕЛЬСТВО ТЕОРЕМЫ 4 основано на привлечении соображений, связанньх с действием операторов Теплища $T_{\bar{h}}$. Достаточно (и это несложно показать, см., например, [18]) установить, что оператор Теплица

$$
\left(T_{\bar{h}} f\right)(z)=\left(\frac{1}{2 \pi}\right) \int_{T} \frac{\overline{h(t)} f(t)}{1-\bar{t} z} d m(t)
$$

действует из $\mathrm{BMOA}_{\alpha, n, q, \beta}(U)$ в $\mathrm{BMOA}_{\alpha, n, q, \beta}(U)$ при $h \in H^{\infty}(U)$.

Заметим, что часть намеченной программы уже вьполнена; если $D^{n} f \in H^{1}(U)$, то $D^{n}\left(T_{\bar{h}} f\right)(z) \in H^{1}(U), n \in \mathbb{N}, h \in H^{\infty}$.

Этот результат приведен в работе [4].

Далее, тригонометрические полиномы, как легко видеть, при $q \leqslant 2, \beta \in(0, \infty)$ или при $q>2, \beta>q-2$ принадлежат пространствам $\mathrm{BMOA}_{\alpha, n, q, \beta}$.

Заметим также, что при $\alpha>n$ конечность "нормы" $\|f\|_{\text {ВмоА }_{\alpha, n, q, \beta}}<\infty$ обеспечивает включение $f^{(n)} \in H^{1}$.

Для доказательства теоремы проведем оценку величины

$$
\left(\int_{T} \frac{\left|\left(\widetilde{D}^{\alpha} F\right)(\xi)-\left(\widetilde{D}^{\alpha} F\right)(z)\right|}{|1-\bar{\xi} z|^{q}} d m(\xi)\right)(1-|z|)^{\beta},
$$

где $z \in U, \alpha \geqslant 0, \beta>0, q \in(0, \infty)$,

$$
F(z)=\left(T_{\bar{h}} f\right)(z), \quad f \text {-полином, } f \in \mathrm{BMOA}_{\alpha, n, q, \beta} .
$$

Для этого нам понадобится следующая оценка. Ее доказательство проводится стандартным образом и содержится, например, в [8]. Имеем

$$
\int_{U} \frac{\left(1-|\xi|^{2}\right)^{s} d m_{2}(\xi)}{|1-\bar{\xi} z|^{r}|1-\bar{\xi} \omega|^{\nu}} \lesssim \frac{C_{1}}{|1-\bar{z} \omega|^{r+\nu-s-2}}
$$


$r-s, \nu-s<2, s>-1, r, \nu \geqslant 0, r+\nu-s>2$.

Нам потребуется также следующее очевидное неравенство:

$$
\left|(1-\xi z)^{\alpha}-(1-\xi \omega)^{\alpha}\right| \leqslant C\left(|1-z \bar{\omega}|\left(|1-\xi z|^{\alpha-1}+|1-z \bar{\omega}|^{\alpha-1}\right)\right)
$$

при $\alpha \geqslant 1, \xi, \omega \in U, z \in T$.

Действительно, легко видеть, что для любой точки $\widetilde{\omega}$, лежащей между $z$ и $\omega$, имеем

$$
\begin{gathered}
f(\widetilde{\omega})=(1-\xi \widetilde{\omega})^{\alpha}, \quad \alpha \geqslant 1, \\
\left|f^{\prime}(\widetilde{\omega})\right| \leqslant \alpha|1-\xi \widetilde{\omega}|^{\alpha-1} \leqslant \alpha\left(|1-\xi z|^{\alpha-1}+|z-\widetilde{\omega}|^{\alpha-1}\right) \leqslant \alpha\left(|1-\xi z|^{\alpha-1}+|1-\bar{z} \omega|^{\alpha-1}\right), \\
\left|(1-\xi z)^{\alpha}-(1-\xi \omega)^{\alpha}\right| \leqslant\left|f^{\prime}(\widetilde{\omega})\right||1-\bar{z} \omega| \lesssim|1-\bar{z} \omega|\left(|1-\bar{z} \omega|^{\alpha-1}+|1-\xi z|^{\alpha-1}\right) .
\end{gathered}
$$

Следовательно,

$\left|(1-z \omega)^{\alpha+1}-(1-z \xi)^{\alpha+1}\right| \lesssim|1-\omega \bar{\xi}|^{\alpha+1}+|1-\omega \bar{\xi}||1-\overline{\xi z}|^{\alpha}, \quad \omega, z \in U, \quad \xi \in T, \quad \alpha \geqslant 0$.

Учитывая тот факт, что $f$ - полином, вьводим $\left(F(\omega)=\left(T_{\bar{\varphi}} f\right)(\omega)\right)$

$$
\begin{aligned}
J(\omega) & =\int_{T} \frac{\left|\widetilde{D}^{\alpha} F(\xi)-\widetilde{D}^{\alpha} F(\omega)\right|}{|1-\xi \bar{\omega}|^{q}} d m(\xi)(1-|\omega|)^{\beta} \lesssim \lim _{R \rightarrow 1} J_{R}(\omega) \\
& =\lim _{R \rightarrow 1}\left(\int_{T}\left|\int_{T} \frac{\overline{\varphi(t)} f(t) d m(t)}{(1-\bar{t} R \xi)^{\alpha+1}}-\int_{T} \frac{\overline{\varphi(t)} f(t) d m(t)}{(1-\bar{t} R \omega)^{\alpha+1}}\right| \frac{(1-|\omega|)^{\beta}}{|1-\bar{\omega} \xi|^{q}} d m(\xi)\right) \\
& =\int_{T}\left|\lim _{R \rightarrow 1} \int_{T} \frac{\overline{\varphi(R t)} f(R t)(1-t R \bar{\omega})^{\alpha+1}-(1-t R \bar{\xi})^{\alpha+1}}{\overline{(1-t R \bar{\xi})^{\alpha+1}(1-t R \bar{\omega})^{\alpha+1}}} d m(t)\right| \times \frac{(1-|\omega|)^{\beta}}{|1-\omega \bar{\xi}|^{q}} d \xi .
\end{aligned}
$$

Учитывая лемму 2 , получим

$$
\begin{aligned}
J(\omega) \lesssim & \int_{T} \int_{U} \frac{|\varphi(z)|\left|\widetilde{D}^{k} f(z)\right|(1-|z|)^{k-1}\left|(1-z \bar{\omega})^{\alpha+1}-(1-z \bar{\xi})^{\alpha+1}\right|}{|1-z \bar{\xi}|^{\alpha+1}|1-z \bar{\omega}|^{\alpha+1}} d m_{2}(z) \\
& \times \frac{(1-|\omega|)^{\beta}}{|1-\bar{\omega} \xi|^{q}} d m(\xi) .
\end{aligned}
$$

Из оценки (8) выводим $J(\omega) \leqslant\left(\sup _{z \in U}|\varphi(z)|\right)\left(J_{1}+J_{2}\right)$.

Оценим каждое слагаемое в отдельности.

Имеем

$$
\begin{aligned}
J_{1} & \lesssim \int_{T} \int_{U} \frac{\left|\widetilde{D}^{k} f(z)\right|(1-|z|)^{k-1}|1-\omega \bar{\xi}|^{\alpha+1}}{|1-z \bar{\xi}|^{\alpha+1}|1-z \bar{\omega}| \alpha+1} \frac{(1-|\omega|)^{\beta}}{|1-\bar{\omega} \xi|^{q}} d m(\xi) d m_{2}(z) \\
& \lesssim\left[\sup _{|z|<1}\left(\left|\widetilde{D}^{k} f(z)\right|(1-|z|)^{\beta}\right)\right] * \widetilde{J}_{1}^{\prime}=\widetilde{J}_{1} * \widetilde{J}_{1}^{\prime}, \quad \alpha \geqslant 0 .
\end{aligned}
$$

Учтем, что

$$
\widetilde{J}_{1}=\sup _{|z|<1}\left|\widetilde{D}^{k} f(z)\right|(1-|z|)^{\beta} \lesssim \sup _{|z|<1}\left|\widetilde{D}^{\alpha} D^{q-1} f(z)\right|(1-|z|)^{\beta}
$$


при $k=\alpha+q-1, q>1, \alpha \geqslant 0$.

Поэтому из интегральной формулы Коши выводим

$$
\widetilde{J}_{1} \lesssim \sup _{|z|<1} \int_{T} \frac{\left|\widetilde{D}^{\alpha} f(\xi)-\widetilde{D}^{\alpha} f(z)\right|}{|1-\bar{\xi} z|^{q}} d m(\xi)(1-|z|)^{\beta},
$$

где $f$ - полином.

Учитывая оценку (7), получаем

$$
\begin{aligned}
\widetilde{J}_{1}^{\prime} & =\int_{T} \int_{U} \frac{(1-|z|)^{k-1-\beta}(1-|\omega|)^{\beta} d m(\xi) d m_{2}(z)}{|1-\bar{\xi} z|^{\alpha+1}|1-z \bar{\omega}|^{\alpha+1}|1-\bar{\omega} \xi|^{-\alpha-1+q}} \\
& \leqslant \int_{T} \int_{U} \frac{(1-|z|)^{\alpha+q-2-\beta}(1-|\omega|)^{\beta} d m(\xi) d m_{2}(z)}{|1-\bar{\xi} z|^{\alpha+1}|1-z \bar{\omega}|^{\alpha+1}|1-\omega \bar{\xi}|^{q-\alpha-1}} \\
& \lesssim\left\{\begin{array}{c}
\int_{T} \frac{(1-|\omega|)^{\beta} d m(\xi)}{|1-\bar{\omega} \xi|^{\beta+1}} \leqslant C \\
\text { при } \beta>0, \quad \beta+1<q<\alpha+\beta+2, \\
\int_{T} \frac{d m(\xi)}{|1-\bar{\omega} \xi|^{q-\alpha-1}}\left(\int_{0}^{1} \frac{(1-|z|)^{q-3-\beta} d|z|}{(1-|z||\omega|)^{\alpha}}\right)(1-|\omega|)^{\beta} \leqslant C \\
\text { при }(\alpha+2, \beta+2)<q<\alpha+\beta+2, \quad \alpha>0 .
\end{array}\right.
\end{aligned}
$$

При получении последней оценки мы учли неравенство $(2)$ и неравенство $|1-\bar{\xi} z|^{\alpha+1} \geqslant$ $(1-|z|)^{\alpha+1}, \alpha>-1$.

Оценим далее слагаемое $J_{2}$. Имеем $(k=\alpha+q-1)$

$$
\begin{aligned}
J_{2} & \lesssim \int_{T} \int_{U} \frac{\left|\widetilde{D}^{k} f(z)\right|(1-|z|)^{k-1}|1-\bar{\omega} \xi||1-\xi \bar{z}|^{\alpha}(1-|\omega|)^{\beta} d m(\xi) d m_{2}(z)}{|1-\xi \bar{z}|^{\alpha+1}|1-z \bar{\omega}|^{\alpha+1}|1-\bar{\omega} \xi|^{q}} \\
& \lesssim \int_{T} \int_{U} \frac{(1-|z|)^{\alpha+q-2-\beta}(1-|\omega|)^{\beta} d m(\xi) d m_{2}(z)}{|1-\bar{\omega} \xi|^{q-1}|1-z \bar{\xi}||1-\bar{z} \omega|^{\alpha+1}}=\widetilde{J}_{2} .
\end{aligned}
$$

Далее, учитьвая оценку (7), выводим

$\int_{U} \frac{(1-|z|)^{\alpha+q-2-\beta} d m_{2}(z)}{|1-z \bar{\omega}|^{1+\alpha}|1-\bar{\xi} z|} \lesssim \frac{1}{|1-\omega \bar{\xi}|^{\beta+2-q}}$ при $q>\beta+1-\alpha, \quad q-2<\beta<q-1$ и поэтому

$$
\widetilde{J}_{2} \lesssim \int_{T} \frac{(1-|\omega|)^{\beta} d m(\xi)}{|1-\bar{\xi} \omega|^{\beta+1}} \leqslant C
$$

или, учитывая оценку $|1-z \bar{\xi}|^{-1} \leqslant(1-|z|)^{-1}$, имеем

$$
\widetilde{J}_{2} \lesssim \int_{0}^{1} \frac{(1-|z|)^{\alpha+q-3-\beta} d|z|}{(1-|z||\omega|)^{\alpha}}(1-|\omega|)^{\beta-q+2} \leqslant C
$$

при $\alpha>0, q>2,-\alpha+\beta+2<q<\beta+2$.

Заметим теперь, что в ходе доказательства теоремы, мы установили, что пространства $\mathrm{BMOA}_{\alpha, n, q, \beta}$ вкладываются в аналитические пространства Бесова

$$
H_{\beta}^{\infty}(U)=\left\{f \in H(U): \sup _{|z|<1}\left|\widetilde{D}^{k} f(z)\right|(1-|z|)^{\beta}<\infty\right\}
$$

при $k=\alpha+q-1$, в которых многочлены образуют всюду плотное множество (см., например, [19]). Поэтому $T_{\bar{\varphi}}$ при $\varphi \in H^{\infty}$ - ограниченный оператор из $H_{\beta}^{\infty}$ в $\mathrm{BMOA}_{\alpha, n, q, \beta}$. Остается еще раз учесть, что $\mathrm{BMOA}_{\alpha, n, q, \beta} \subset H_{\beta}^{\infty}$. Теорема 4 доказана. 
ЗАмЕчАниЕ 8. Вышеустановленная теорема 4 распространяет результат о делении в классах $\mathrm{BMOA}_{n}, n \in \mathbb{N}$, сразу в нескольких направлениях. Рассмотрим, например, случай $\beta=1, \alpha=n \in \mathbb{N}$. Тогда $\mathrm{BMOA}_{n, n, q, 1}=\mathrm{BMOA}_{n, q}$ и деление на внутренний множитель в этих классах (и об этом было упомянуто вьше) было установлено в [4] при $q=2$.

Из теоремы 4 следует, к примеру, что это верно также при $2<q<3$ (см. первую группу неравенств в условиях теоремы).

\section{3. Доказательство вспомогательных утверждений.}

Лемма 1. Справедливь следующие утверэлдения:

1) имеем

$$
\sup _{|z|<1}\left|D^{k} g(z)\right|(1-|z|)^{k-s} \lesssim\|g\|_{F_{s}^{\infty, q}}, \quad k>s, \quad s \in \mathbb{R}, \quad q \in(0, \infty) ;
$$

2) имеем

$$
\left(\int_{0}^{1} M_{s}(G, R)(1-R)^{\alpha} d R\right)^{p} \lesssim \int_{0}^{1}\left(M_{s}(G, R)\right)^{p}(1-R)^{\alpha p+p-1} d R
$$

$p \leqslant 1, \quad \alpha>-1, \quad G \in H(U), \quad s \in(0, \infty]$

3) функиия $g, g(z)=\sum_{k \geqslant 0} z^{k} /(k+1)$, лехит в пространстве $H_{p-1}^{q, \infty,(p-1) / q}$, $1 / p+1 / q=1, \quad p>1$

4) имеем

$$
\left(\int_{U}|f(z)|(1-|z|)^{\alpha} d m_{2}(z)\right)^{p} \lesssim \int_{U}|f(z)|^{p}(1-|z|)^{\alpha p+2 p-2} d m_{2}(z),
$$

$0<p \leqslant 1, \quad f \in H(U),(\alpha+2) p>1$.

ДоказАТЕЛЬСтво. Оценка (9) установлена в [7], неравенство из п. 4 в [16]. Докажем вторую оценку в лемме.

Имеем

$$
\begin{aligned}
\left(\int_{0}^{1} M_{s}(G, R)(1-R)^{\alpha} d R\right)^{p} & \lesssim \sum_{k \geqslant 0}\left(\int_{1-2^{-k}}^{1-2^{-k-1}}\left(M_{s}(G, R)\right) d R\right)^{p} 2^{-k \alpha p} \\
& \lesssim \sum_{k \geqslant 0}\left(M_{s}\left(G, 1-2^{-k-1}\right)\right)^{p} 2^{-k p(\alpha+1)} \\
& \lesssim \sum_{k \geqslant 0}\left(\int_{1-2^{-k-1}}^{1-2^{-k-2}}\left(M_{s}(G, r)\right)^{p} d r\right) 2^{-k(\alpha p+p)} 2^{k} \\
& \lesssim \int_{0}^{1}\left(M_{s}(G, r)\right)^{p}(1-r)^{\alpha p+p-1} d r .
\end{aligned}
$$

Покажем справедливость утверждения, содержащегося в п. 3) вьшеприведенной леммы.

Имеем

$$
\begin{aligned}
\|g\|_{H_{p-1}^{q, \infty}, \frac{p-1}{q}} & =\sup _{r} M_{q}\left(D^{p-1} g, r\right)(1-r)^{\frac{p-1}{q}} \lesssim(z=r \xi) \\
& \lesssim\left(\sup _{r} \int_{T}\left|\frac{1}{(1-z)^{(p-1) q}}\right| d m(\xi)\right)^{\frac{1}{q}}(1-r)^{\frac{p-1}{q}} \lesssim \text { const. }
\end{aligned}
$$

Лемма доказана. 
Лемма 2. 1) Пусть $\alpha>0, f, g \in H(U) ;$ тогда

$$
\begin{aligned}
\left|\int_{T} f(r t) \overline{g(r t)} d m(t)\right| \leqslant C(\alpha) \int_{U}|f(\omega)|\left|\widetilde{D}^{\alpha} g(\omega)\right|(1-|\omega|)^{\alpha-1} d m_{2}(\omega), & \frac{1}{2}<r<1, \\
\left|\int_{T} f(r t) g(r \bar{t}) d m(t)\right| \leqslant C(\alpha) \int_{U}|f(\omega)|\left|D^{\alpha} g(\bar{\omega})\right|(1-|\omega|)^{\alpha-1} d m_{2}(\omega), & \frac{1}{2}<r<1 .
\end{aligned}
$$

2) Ecли $D^{\gamma} f \in H_{0}^{p, q, \alpha}$, mo $\widetilde{D}^{\gamma} f \in H_{0}^{p, q, \alpha}, \quad 0<p, q \leqslant \infty, \quad \gamma \in \mathbb{R}, \quad \alpha>0$, причем справедливо и обратное утверждение.

3) Имеет место оценка

$$
M=\int_{T} \frac{d m(\xi)}{|1-\bar{z} \xi|^{\gamma_{1}}|1-\omega \bar{\xi}|^{\gamma_{2}}} \leqslant \frac{C_{1}(1-|\omega|)^{1-\gamma_{2}}}{|1-z \bar{\omega}|^{\gamma_{1}}}+\frac{C_{2}(1-|z|)^{1-\gamma_{1}}}{|1-z \bar{\omega}|^{\gamma_{2}}}
$$

$\gamma_{1}, \gamma_{2}>1, \quad z, \omega \in U$.

ДокАЗАТЕЛЬСтво. Оценки в п. 1) следствия равенств

$$
\begin{aligned}
\frac{1}{2 \pi} \int_{T} f(r \xi) \overline{g(r \xi)} d m(\xi)= & \left(\frac{2^{\alpha-1}}{r^{2}(\alpha-1) ! \pi}\right) \\
& \times \int_{0}^{r} \int_{0}^{2 \pi} f(\omega) \overline{D^{\alpha} g(\omega)}\left(\log \frac{r}{|\omega|}\right)^{\alpha-1} d m_{2}(\omega), \quad \alpha>1 \\
\frac{1}{2 \pi} \int_{T} f(r \xi) g(r \bar{\xi}) d m(\xi)= & r^{-2(\alpha+2)}\left(\frac{\alpha+2}{\pi}\right) \int_{0}^{r} \int_{-\pi}^{\pi} g(\rho \bar{\xi}) D^{\alpha+2} f(\rho \xi) \\
& \times\left(r^{2}-\rho^{2}\right)^{\alpha+1} \rho d \rho d m(\varphi), \quad \alpha>-2
\end{aligned}
$$

которые легко можно проверить почленньм интегрированием (см., например, [6], [11]).

2) Это утверждение установлено в [15].

3) Имеем

$$
\bar{M}=\int_{\xi:|1-\bar{\xi} z| \geqslant \sigma|1-z \bar{\omega}|}(\ldots) d m(\xi)+\int_{\xi:|1-\xi \bar{z}|<\sigma|1-z \bar{\omega}|}(\ldots) d m(\xi)=\bar{M}_{1}+\bar{M}_{2}
$$

Далее, легко видеть, что если число $\sigma$ выбрать достаточно близким к нулю, то в силу неравенства $|1-\xi \bar{z}|<\sigma|1-z \bar{\omega}|$ будем иметь $C_{2}|1-\omega \bar{z}|<|1-\omega \bar{\xi}|<C_{1}|1-\omega \bar{z}|, \xi \in T$, $\omega, z \in U$.

Поэтому

$$
\bar{M}_{1} \leqslant C_{3} \frac{(1-|\omega|)^{1-\gamma_{2}}}{|1-\omega \bar{z}|^{\gamma_{1}}}, \quad \bar{M}_{2} \leqslant C_{4} \frac{(1-|z|)^{1-\gamma_{1}}}{|1-\omega \bar{z}|^{\gamma_{2}}} .
$$

Лемма 2 доказана. 


\section{СПИСОК ЦИТИРОВАННОЙ ЛИТЕРАТУРЫ}

[1] Mateleviĉ M., Pavloviĉ M. Multipliers of $H^{p}$ and BMOA // Pacific J. Math. 1990. V. 146. P. 71-84.

[2] Kehe Zhu. Duality of Bloch spaces and norm convergence of Taylor series // Michigan Math. J. 1991. V. 38. P. 89-101.

[3] Zabulionis A. On the differential operator in the analytic function spaces // Litovsk. Mat. Sb. 1984. V. 24. №1. P. 53-59.

[4] Шамоян Ф.А. Теплицевы операторы в некоторых пространствах голоморфных функций и новая характеристика классов ВМОА // Изв. АН. АрмССР. 1987. № 2. С. 122-132.

[5] Гарнетт Д. ЖК. Ограниченные аналитические функции. М.: Мир, 1984.

[6] Шамоян Р. Ф. Обобщенное преобразование Харди и операторы Теплица в пространствах типа ВМОА // Укр. матем. ж. 2001. Т. 53. №9. С. 1260-1271.

[7] Ortega J. M., Fabrega J. Hardy inequality and embeddings in holomorphic Triebel-Lizorkin spaces // Illinois Math. J. 1999. V. 43. № 4. P. 733-751.

[8] Ortega J. M., Fabrega J. Pointwise multipliers and corona-type decomposition in BMOA // Ann Inst. Four. Gren. 1996. V. 46. № 1. P. 111-137.

[9] Шамоян Р.Ф. Мультипликаторы степенных рядов, операторы Теплица и двойственность в некоторых пространствах голоморфных в поликруге функций. Дисс. ... канд. физ.-матем. наук. М., 2001.

[10] Трибель Х. Теория функциональных пространств. М.: Мир, 1986.

[11] Шамоян Р. Ф. О мультипликаторах из пространств типа Бергмана в пространства Харди в поликруге // Укр. матем. ж. 2000. Т. 10. С. 405-415.

[12] Шамоян Р. Ф. Мультипликаторы степенных рядов, операторы Теплица и теоретико-множественные вложения пространств Харди-Соболева-Джрбашяна // Изв. АН Респ. Армения. 1999. № 4. С. 43-59.

[13] Шведенко С. В.Классы Харди и связанные с ними пространства аналитических функций в единичном круге и шаре // Итоги науки и техники. Т. 23. Сер. матем. анализ. М., 1985. C. $3-123$.

[14] Шамоян Р. Ф. О характеристиках типа ВМО одного класса голоморфньгх в круге функций // Сиб. матем. ж. 2002 (принято к печати).

[15] Buckley S.M., Koskela P., Vukotiĉ D. Fractional integration, differentiation, and weighted Bergman spaces // Proc. Cambridge Philos. Soc. 1999. № 2. P. 377-385.

[16] Djrbashian A., Shamoyan F. Topics in the theory of $A_{\alpha}^{p}$ spaces // Teubner Texte zur Math. 1988. V. 105. P. 1-199.

[17] Yevtiĉ M., Pavloviĉ M. Coefficient multipliers on spaces of analytic functions // Acta Sci. Math. 1998. V. 64. P. 531-545.

[18] Shirokov N. K. Analytic Functions Smooth up to the Boundary // Lecture Notes in Math.: Springer, 1988.

[19] Janson S., Petre J. Semmes, Hankel, and Toeplitz operators on some spaces of analytic functions // Duke Math. J. 1983. P. 936-957.

[20] Aleksandrov A.B., Peller V. V. Hankel operators and similarity to a contraction // Intern. Math. Res. Notes. 1996. №9. P. 263-275. 